\title{
Alpelisib After Treatment with CDK4/6i and Aromatase Inhibitor: Case Report
}

\section{Agnieszka Mlodzinska | Agnieszka Jagiello-Gruszfeld*}

*Correspondence: Agnieszka Jagiello-Gruszfeld

Address: Breast Cancer and Reconstructive Surgery Department, Maria Sklodowska-Curie National Research Institute of Oncology, Warsaw, Poland

e-mail $\bowtie$ : agnieszka.jagiellogruszfeld@gmail.com

Received: 26 November 2021; Accepted: 03 December 2021

Copyright: (C) 2021 Jagiello-Gruszfeld A. This is an open-access article distributed under the terms of the Creative Commons Attribution License, which permits unrestricted use, distribution, and reproduction in any medium, provided that the original work is properly cited.

\section{ABSTRACT}

Alpelisib is an oral drug, a phosphatidylinositol 3 kinase inhibitor (PI3K) which plays a fundamental role in the cancer process. In May 2019 Alpelisib in combination with fulvestrant was approved by FDA in treatment of PIK3CA-mutated, hormone receptor-positive, human epidermal growth factor receptor2-negative advanced breast cancer. Since 2020 the drug has also recommendation of European Medicines Agency (EMA). The following is a case of a patient with primary metastatic breast cancer who was treated with alpelisib in combination with fulvestrant after prior chemotherapy and an aromatase inhibitor with ribociclib, obtaining a significant clinical benefit.

Keywords: Metastatic Breast Cancer, Alpelisib, PIK3CA Mutation

\section{Introduction}

Approximately $40 \%$ of patients with hormone receptor-positive, HER -2-negative advanced breast cancer have PIK3CA-mutated tumors, which have been identified with resistance to hormone therapy (Fusco et al., 2021). In SOLAR-1 clinical trial alpelisib - an $\alpha$-selective phosphatidylinositol-3-kinase inhibitor - combined with fulvestrant showed significantly improved progression-free survival (PFS). In BYLieve study same drugs combination demonstrated clinical efficacy (André et al., 2019; Rugo et al., 2021). There are limitations in the data comparing efficiency of alpelisib with fulvestrant to the standard therapy used after CDK4/6i treatment. To date, few reports have been published on the efficacy of treatment with alpelisib in combination with fulvestrant in patients who have previously received chemotherapy and CDK4 /6i. The patient's case described below shows that patients in a similar situation benefit from alpelisib therapy. 


\section{Case Presentation}

The 60-year-old patient noticed swelling of the left eyelid in February 2019, she was diagnosed in the provincial hospital, where the possibility of infiltrative inflammatory changes was found. In addition, due to the increased level of creatinine, an ultrasound of the abdominal cavity was performed, which revealed a heterogeneous area of $45 \times 35 \times 40 \mathrm{~mm}$ in the left lobe of the liver and bilateral dilatation of the renal-pelvic system caused by a tumor infiltrate in the retroperitoneal region. Left-sided nephrostomy was performed. There was also anemia (HGB $<7.5 \mathrm{G} / \mathrm{dL}$ ) due to haematuria. $3 \mathrm{UBC}$ units were transfused. Oncological diagnosis was not performed.

In April 2019, she reported for her first visit to the National Institute of Oncology in Warsaw due to suspicion of cancer from an unknown origin. The patient reported weight loss (approx. $4 \mathrm{~kg}$ in the one month), diarrhea and periodic abdominal pain. Good general condition - ECOG 1. Physical examination revealed an infiltration involving the outer quadrants of the right breast. Therefore, a biopsy of the lesion in the right breast was performed: G1 lobular carcinoma, ER-100\%, PGR-90\%, HER2-negative, Ki67-10\%.

CT scan that showed cervical and axillary lymphadenopathy, two lesions in the liver, right-sided hydronephrosis, suspiciously enlarged both ovaries, free fluid in the peritoneal cavity, thickened wall of the stomach, peritoneal infiltration and mixed changes in the skeleton with a predominance of lytic lesions in the iliac and sacral bones, left scapula, C5 vertebra with pathological fracture of its shaft (after neurosurgical consultation without urgent radiotherapy). The patient also underwent gastroscopy, that showed an abnormal mucosa, the biopsy of which result as a metastasis of breast cancer. Fine-needle biopsy of the cervical and axillary lymph nodes also confirmed metastatic cancer with the most likely origin from the breast. Due to the reported pain of the knee joint, an X-ray was performed - osteolytic changes were found in both the proximal and distal parts of the femur, with no risk of fracture.

After a multidisciplinary council, she was qualified for palliative chemotherapy due to visceral crisis, She received doxorubicin administered weekly in a dose of $20 \mathrm{mg} / \mathrm{m} 2$ (metronomic treatment) chemotherapy was started only in June 2019 due to various problems with the timing of tests and visits. . She also received supportive treatment with zoledronic acid and darbopoetin alfa due to anemia during chemotherapy. In control CTs in August and December 2019, the disease stabilized. The patient continued treatment until March 2020, she received a total of 35 treatment courses until the lifetime dose of anthracyclines was exhausted. Then maintenance therapy with Tamoxifen was started. In follow-up CT in August 2020, the disease progressed in the liver. She received ribociclib 1 x $600 \mathrm{mg}$ for 21 days, followed by 7 breaks and letrozole $1 \times 2.5 \mathrm{mg}$ daily (from 01.Sep.2020), as well as denosumab due to worsening of kidney function. The patient also underwent palliative radiotherapy on the left hip area (2000 cGy /g in 5 
fractions). A control CT scan from February 2021 revealed disease progression - interstitial changes in the lungs, enlargement of metastatic changes in the liver, increased urinary retention in the right kidney. A genetic test was performed to confirm the presence of a PiK3CA gene mutation in the neoplastic tissue, which allowed the patient to be treated with alpelisib. From March 2021, the patient receives alpelisib (Piqray ${ }^{\circledR}$ ) at the recommended dose, i.e. $300 \mathrm{mg} /$ day orally (i.e. 2 tablets of $150 \mathrm{mg}$ ) in the morning, immediately after breakfast, in combination with hormone therapy with fulvestrant $500 \mathrm{mg}$ i.m. every 4 weeks (in the first cycle, $500 \mathrm{mg}$ was also given on day 15). The disease was stabilized in CT examinations performed every 3 months. Currently, the patient has been continuing treatment for over 9 months with very good tolerance, and did not require any dose modification of alpelisib dose.

In the first 4 weeks of treatment were observed grade 2 skin lesions. Symptomatic corticosteroid cream and treatment with bilastine $20 \mathrm{mg}$ / day were applied. After 4 weeks, the changes subsided. After about 3 weeks, the patient noticed grade 1 hair loss, which could be caused by alpelisib treatment. There was no treatment in this case.

Surprisingly, in our case, the most frequently reported side effect of alpelisib, i.e. hyperglycaemia, was not found. Fasting blood glucose levels were determined prior to initiation of treatment and weekly thereafter for the first 8 weeks of treatment, when the risk of developing an elevated blood glucose level is highest. Subsequently, blood glucose levels were assessed every 4 weeks. HbA1c levels, as recommended, were assessed after 4 weeks of treatment, and then every 3 months, also without any abnormalities.

There was also no increase in creatinine levels, which, taking into account the earlier course of the disease and baseline creatinine levels, was a concern before starting the therapy.

\section{Discussion}

Alpelisib is a class I $\alpha$-specific inhibitor of phosphatidylinositol-3-kinase. Inhibition of PI3K by alpelisib has been shown to increase estrogen receptor (ER) transcription in breast cancer cells. The PI3K / AKT signaling pathway is responsible for glucose homeostasis, and hyperglycemia is a predicted adverse reaction resulting from the inhibition of PI3K activity associated with the proper molecular target of the inhibitor (Copur, 2020; Porta et al., 2014).

Alpelisib has been studied in a phase III randomized, double-blind study of alpelisib in combination with fulvestrant in postmenopausal women and men with known hormone-dependent HER2-negative breast cancer who had progressed on aromatase inhibitor-based therapy (when used in combination with a CDK4 / 6 inhibitor or without a CDK4 / 6 inhibitor) or afterwards (Ciruelos et al., 2021; Turner et 
al., 2021; Wilhoit et al., 2020). Based on efficacy results in the PIK3CA mutation cohort, a statistically significant increase in time to disease progression (PFS) was demonstrated in patients receiving alpelisib plus fulvestrant compared to patients receiving placebo plus fulvestrant with an estimated reduction in the risk of disease progression or death by 35\% (Andre et al., 2021). The SOLAR-1 study showed a statistically significant difference between the alpelisib plus fulvestrant and fulvestrant plus placebo groups in progression-free survival (median progression-free survival was 11.0 months in the alpelisib group, compared to 5.7 months in the placebo group) (Andre et al., 2019), as well as in terms of response rate $(26.6 \%$ responders in the alpelisib group compared to $12.8 \%$ patients in the placebo group) and for clinical benefit, defined as ongoing CR, PR or SD at least 24 weeks. A clinical benefit was seen in $61.5 \%$ of patients in the alpelisib group and $45.3 \%$ of patients in the placebo group. There was no statistically significant difference between the groups in terms of overall survival. The median OS for the PIK3CAmutant group was 39.3 months for alpelisib plus fulvestrant and 31.4 months for fulvestrant plus placebo (hazard ratio $(\mathrm{HR})=0.86(95 \% \mathrm{CI}, 0.64-1.15 ; \mathrm{P}=0.15)$ (Andre et al., 2021) Median OS in patients with lung and / or liver metastases was 37.2 months and 22.8 months in the alpelisib and placebo groups, respectively (HR = $0.68(0.46-1.00) 95 \% \mathrm{CI})$ (Andre et al., 2021).

In the BYLieve study (clinical trial without a control group) in a cohort of patients treated with a CDK4 / 6 inhibitor in combination with an aromatase inhibitor, the median PFS was 7.3 months, the median OS was 17.3 months, and the overall response rate was 17\% (Rugo et al., 2021).

The Turner publication from 2021 comparing the results of the BYLive study with the data from real word data showed a statistically significantly higher median PFS in the population of patients receiving alepelisib (patients from the BYLieve study) compared to the RDW population (Turner et al., 2021).

In the SOLAR-1 study, the use of alpelesib in combination with fulvestrant was associated with a statistically significant reduction in quality of life (according to EORTC QLQ-C30) relative to baseline, especially due to loss of appetite and diarrhea compared to the fulvestrant group. Grade 3 and 4 treatment-related adverse events were reported six times more frequently in the alpelesib plus fulvestrant arm compared to placebo plus fulvestrant (66.9\% vs 11.8\%) (Ciruelos et al., 2021)

The most common adverse reactions reported in clinical trials were increased glucose level (79.2\%), increased creatinine (67.6\%), diarrhea (59.5\%), increased gammaglutamyltranspeptidase (53.2\%), rash (51.8\%), lymphocyte count decreased (55.3\%), nausea (46.8\%), increased alanine aminotransferase (44.0\%), anemia (44.0\%), fatigue (43.3\%), increased lipase (42.6\%), decreased appetite $(35.9 \%)$, stomatitis (30.3\%), vomiting (28.5\%), weight loss (27.8\%), hypocalcaemia (27.8\%), 
decrease glucose level (26.8\%), prolongation of activated partial thromboplastin time (aPTT) (22.2\%) and alopecia (20.4\%) [1].

In the majority of cases of minor or moderate complications, there is no reason to modify the treatment plan, however, if necessary, a temporary interruption of alpelisib administration and dose reduction are envisaged. The first reduction of the dose of the drug provides for its reduction to $250 \mathrm{mg} /$ day (1 tablet of $200 \mathrm{mg}+1$ tablet of $50 \mathrm{mg}$ ), the subsequent reduction of the dose leads to the administration of alpelisib at a dose of $200 \mathrm{mg} /$ day (1 tablet of $200 \mathrm{mg}$ ). A maximum of 2 -fold dose reduction is recommended, followed by permanent treatment discontinuation.

Baseline type II diabetes mellitus (type I diabetes mellitus is a contraindication for the inclusion of alpelisib), pre-diabetes mellitus, baseline $\mathrm{BMI} \geq 30$, and patient age $\geq 75$ years are risk factors for hyperglycaemia in alpelisib-treated patients (Ellis and Ma, 2019; Bertho, 2021).

In the registration trial of alpelisib, a small percentage of patients had to discontinue therapy due to adverse events. The most common adverse reactions leading to discontinuation of treatment were hyperglycaemia (6.3\%), rash (4.2\%), diarrhea (2.8\%) and fatigue (2.5\%) (Ellis and Ma, 2019; Bertho, 2021).

In the case described above, no serious side effects were observed. The patient has been receiving alpelisib therapy for over 9 months with disease stabilization and does not require alpelisib dose adjustment.

\section{References}

André F, Ciruelos E, Rubovszky G, Campone M, Loibl S, Rugo HS, Iwata H, Conte P, Mayer IA, Kaufman B, Yamashita T, Lu YS, Inoue K, Takahashi M, Pápai Z, Longin AS, Mills D, Wilke C, Hirawat S, Juric D; SOLAR-1 Study Group. Alpelisib for PIK3CAMutated, Hormone Receptor-Positive Advanced Breast Cancer. N Engl J Med 2019; 380: 1929-1940.

André F, Ciruelos EM, Juric D, Loibl S, Campone M, Mayer IA, Rubovszky G, Yamashita T, Kaufman B, Lu YS, Inoue K, Pápai Z, Takahashi M, Ghaznawi F, Mills D, Kaper M, Miller M, Conte PF, Iwata H, Rugo HS. Alpelisib plus fulvestrant for PIK3CAmutated, hormone receptor-positive, human epidermal growth factor receptor-2-negative advanced breast cancer: final overall survival results from SOLAR-1. Ann Oncol 2021; 32: 208-217.

Bertho M, Patsouris A, Augereau P, Robert M, Frenel JS, Blonz C, Campone M. A pharmacokinetic evaluation of alpelisib for the treatment of HR+, HER2-negative, PIK3CA-mutated advanced or metastatic breast cancer. Expert Opin Drug Metab Toxicol 2021; 17: 139-152.

Ciruelos EM, Rugo HS, Mayer IA, Levy C, Forget F, Delgado Mingorance JI, Safra T, Masuda N, Park YH, Juric D, Conte P, Campone M, Loibl S, Iwata H, Zhou X, Park J, Ridolfi A, Lorenzo I, André F. Patient-Reported Outcomes in Patients with PIK3CAMutated Hormone Receptor-Positive, Human Epidermal Growth Factor Receptor 2-Negative Advanced Breast Cancer From SOLAR-1. J Clin Oncol 2021; 39: 2005-2015.

Copur MS. Alpelisib to treat breast cancer. Drugs Today (Barc) 2020; 56: 357-363. 
Ellis H and Ma CX. PI3K inhibitors in breast cancer therapy. Curr Oncol Rep 2019; 21: 1-9.

Fusco N, Malapelle U, Fassan M, Marchiò C, Buglioni S, Zupo S, Criscitiello C, Vigneri P, Dei Tos AP, Maiorano E, Viale G. PIK3CA Mutations as a Molecular Target for Hormone Receptor-Positive, HER2-Negative Metastatic Breast Cancer. Front Oncol 2021; $11: 644737$.

Porta C, Paglino C, Mosca A. Targeting PI3K/Akt/mTOR Signaling in Cancer. Front Oncol 2014; 4: 64.

Rugo HS, Lerebours F, Ciruelos E, Drullinsky P, Ruiz-Borrego M, Neven P, Park YH, Prat A, Bachelot T, Juric D, Turner N, Sophos N, Zarate JP, Arce C, Shen YM, Turner S, Kanakamedala H, Hsu WC, Chia S. Alpelisib plus fulvestrant in PIK3CA-mutated, hormone receptor-positive advanced breast cancer after a CDK4/6 inhibitor (BYLieve): one cohort of a phase 2, multicentre, open-label, non-comparative study. Lancet Oncol 2021; 22: 489-498.

Turner S, Chia S, Kanakamedala H, Hsu WC, Park J, Chandiwana D, Ridolfi A, Yu CL, Zarate JP, Rugo HS. Effectiveness of Alpelisib + Fulvestrant Compared with Real-World Standard Treatment Among Patients with HR+, HER2-, PIK3CA-Mutated Breast Cancer. Oncologist 2021; 26: e1133-e1142.

Wilhoit T, Patrick JM, May MB. Alpelisib: A Novel Therapy for Patients with PIK3CA-Mutated Metastatic Breast Cancer. J Adv Pract Oncol 2020; 11: 768-775.

1. https://ec.europa.eu/health/documents/community-register/2020/20200727148490/anx_148490_pl.pdf 